\title{
Supplementary Report on Experimental Autumn Crocus (Colchicum autumnale L.) Poisoning in Cattle: Morphological Evidence of Apoptosis
}

\author{
Manabu YAMADA, Takane MATSUI, Yoshiyasu KOBAYASHI, Hidefumi FURUOKA, Makoto HARITANI ${ }^{1)}$, \\ Masaru KOBAYASHI ${ }^{2)}$ and Michio NAKAGAWA \\ Department of Veterinary Pathology, Obihiro University of Agriculture and Veterinary Medicine, Obihiro, Hokkaido 080-8555, \\ ${ }^{1)}$ Tohoku Branch Laboratory, National Institute of Animal Health, Shichinohe, Kamikita-gun, Aomori 039-2586, and ${ }^{2)}$ National Institute \\ of Animal Health, 3-1-1 Kannondai, Tsukuba, Ibaraki 305-0856, Japan \\ (Received 15 December 1998/Accepted 9 February 1999)
}

ABSTRACT. Previously we reported that tissue destruction characterized by the presence of karyopyknotic, karyorrhectic and mitotically arrested cells was seen in alimentary epithelial cells and lymphocytes in the lymphoid and hemopoietic systems of cattle experimentally administered with autumn crocus (Colchicum autumnale L.). This report deals with the mechanism of acute cellular injury following experimental autumn crocus poisoning in cattle as demonstrated by the in situ DNA strand break analysis and electron microscopy. The analyses revealed that cellular injury caused by autumn crocus was closely associated with apoptosis.—KEY wORDS: apoptosis, autumn crocus (Colchicum autumnale L.), cattle.

Autumn crocus (Colchicum autumnale L.) is a perennial plant classified as a member of the Liliaceae family [4]. Acute poisoning of cattle by autumn crocus is characterized by acute lethal diarrhea caused by ingredient alkaloid colchicine [7]. In a previous study on cattle experimentally intoxicated with autumn crocus, we observed tissue destruction characterized by the presence of karyopyknotic, karyorrhectic and mitotically arrested cells in alimentary epithelial cells and lymphocytes in the lymphoid and hemopoietic systems [17]. Karyopyknosis and karyorrhexis have been regarded as the characteristic morphological evidence of apoptosis, which is an active process of cellular self-destruction accompanied by an internucleosomal DNA fragmentation $[12,16]$.

Although it has been reported that ingredient colchicine induced apoptosis in vitro $[1,15]$, detailed cytotoxic injury of colchicine in the digestive tract or lymphoid and hemopoietic tissues is not clearly understood in vivo $[3,6$, $8,11,13]$.

In order to confirm whether apoptosis is a contributory factor to the cytotoxic injury caused by autumn crocus or not, the present study focuses on the in situ DNA strand break analysis and ultrastructural examination of the digestive and hemopoietic systems from cattle which had been administered with autumn crocus in our previous study [17].

The materials examined were extracted from our previous study of experimental acute autumn crocus poisoning in cattle [17]. Briefly, 4 Holstein-Friesian calves (4-monthold), which had received orally dehydrated bulb powder of autumn crocus ( $4 \mathrm{~g} / \mathrm{kg}$ of body weight) along with assorted feed, were chosen for the present study. Of these, 2 calves were euthanized 20 and $30 \mathrm{hr}$ after administration and the remaining 2 died 48 and $49 \mathrm{hr}$ after administration, respectively. All these calves developed serous diarrhea with hemorrhage and grossly had diffuse intestinal hemorrhage. Histopathological lesions of these animals were tissue destruction showing karyopyknosis, karyorrhexis and mitotically arrested cells in the alimentary epithelium and lymphocytes in the lymphoid and hemopoietic systems.

To investigate the presence of cells with DNA strand break, which is a characteristic finding for the apoptotic process, paraffin embedded specimens of the tongue, forestomach, small intestine and spleen from these calves were examined by the terminal deoxynucleotidyl transferase (TdT)-mediated dUTP-nick end labeling (TUNEL) procedure [5]. The reagents used were obtained from the Apoptag kit (Oncor, Gaithersburg, MD). In addition, representative specimens from a 6-month-old calf without any history of autumn crocus intoxication served as control.

For ultrastructural examination, small pieces of the tongue, forestomach, small intestine and spleen from all calves were fixed in $4 \%$ paraformaldehyde $/ 0.1 \mathrm{M}$ phosphate buffer, postfixed in osmium tetroxide and embedded routinely in resin. Ultrathin sections were stained with saturated uranyl acetate and lead citrate and examined with an H-7500 electron microscope (HITACHI Co., Ltd., Tokyo) at $80 \mathrm{kV}$.

In the small intestine of the control calf, a small number of TUNEL-positive cells were observed only in the upper region of the intestinal villi and there were no positively stained cells in the crypt (Fig. 1a). In contrast, positive cells were numerous involving intestinal epithelia in both crypt and atrophied villi of the intoxicated calves (Fig. 1b). These positively stained cells appeared to be consistent with the cells having karyopyknotic or karyorrhectic nuclei observed by light microscopy. On the other hand, cells undergoing arrested mitosis showed a negative reaction. In the lamina propria and lymph nodules of the small intestine, lymphocytes with a positive reaction were also observed. In the tongue and forestomach of the intoxicated calves, positive reactions were markedly enhanced in comparison with the control calf, and positive cells were distributed in the basal layer of the epithelium. In the spleen of the 


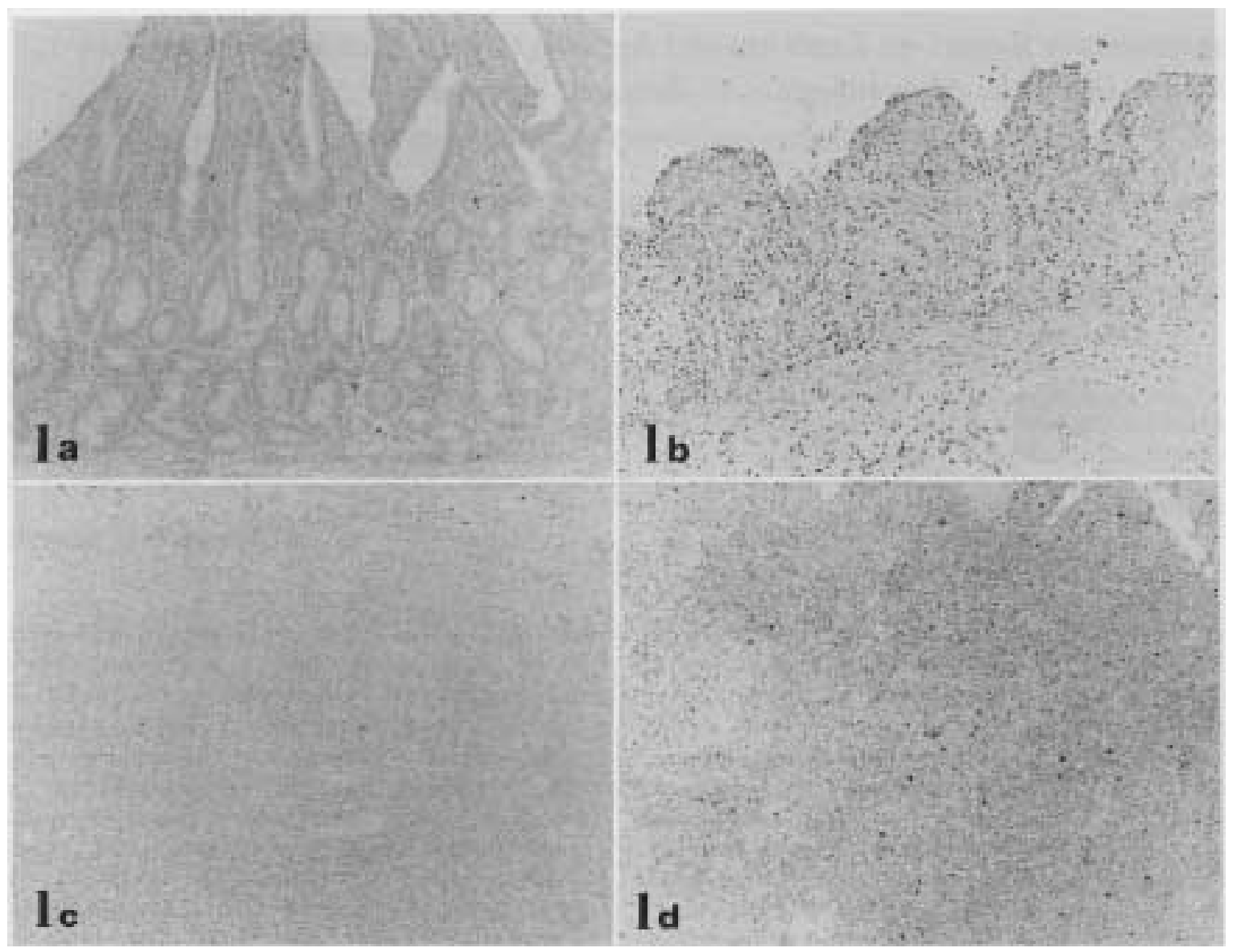

Figs. 1a-d. Comparison of the results of histochemical staining of DNA fragmentation from the intoxicated calf euthanized $30 \mathrm{hr}$ after administration with the control calf. Small intestine (a \& b). Spleen (c \& d). $\times 85$. In the intoxicated calf, positive reactions are markedly enhanced (b) in comparison with control calf (a). Note positively stained cells are widely distributed from the basal to upper regions in the atrophied intestinal villi (b), in contrast positive reactions are confined to the upper region of the epithelial layer of the small intestine of normal calf (a). The spleen of the intoxicated calf, positive reactions are markedly enhanced (d) in comparison with control calf (c).

intoxicated calves, positive reactions were more prominent than those of the control calf (Figs. 1c, d). These positively stained cells appeared to correspond to the karyorrhectic cells in the germinal center and the karyopyknotic cells scattered in the red pulp, as observed by light microscopy. The positive reactions were enhanced in the course of time after administration.

Ultrastructurally, cells having condensed chromatin masses were observed in the intestinal epithelium, in both crypt and atrophied villi of all intoxicated calves (Fig. 2a). The chromatin masses were arranged along the nuclear envelope as homogeneous masses and often assumed a halfmoon configuration just beneath the intact nuclear envelope. In the basal layer of the forestomach and tongue of all intoxicated calves, similar changes were also seen. In the spleen of all intoxicated calves, many lymphocytes having condensed chromatin masses abutting on the nuclear envelope were observed. The lymphocytes showing similar changes were also observed in the lamina propria and lymph nodules of the small intestine.
Mitotically arrested cells, which have swollen cytoplasm and located in the intestinal crypt epithelium, also had condensed nuclear chromatin masses. In addition, marked dilation of endoplasmic reticulum was observed in these cells without mitochondrial abnormality (Fig. 2b). These cells were observed in the basal layer of the tongue and forestomach of the intoxicated calves, but not in the lymphoid and hemopoietic tissues.

In the present study, TUNEL-positive cells are conspicuously found in the epithelium of the digestive tract, and in the lymphoid and hemopoietic tissues. Ultrastructurally, in the organs of the intoxicated calves, the nuclei frequently showed condensation of chromatin along the nuclear envelope. These findings are similar to those of apoptosis [12, 16], suggesting that apoptosis is a contributory factor to lesion in autumn crocus poisoning.

Acute poisoning of cattle by autumn crocus was characterized by acute lethal diarrhea caused by ingredient alkaloid colchicine [7]. Colchicine has been classified into the category of microtubule disrupting drugs (MDD) and 


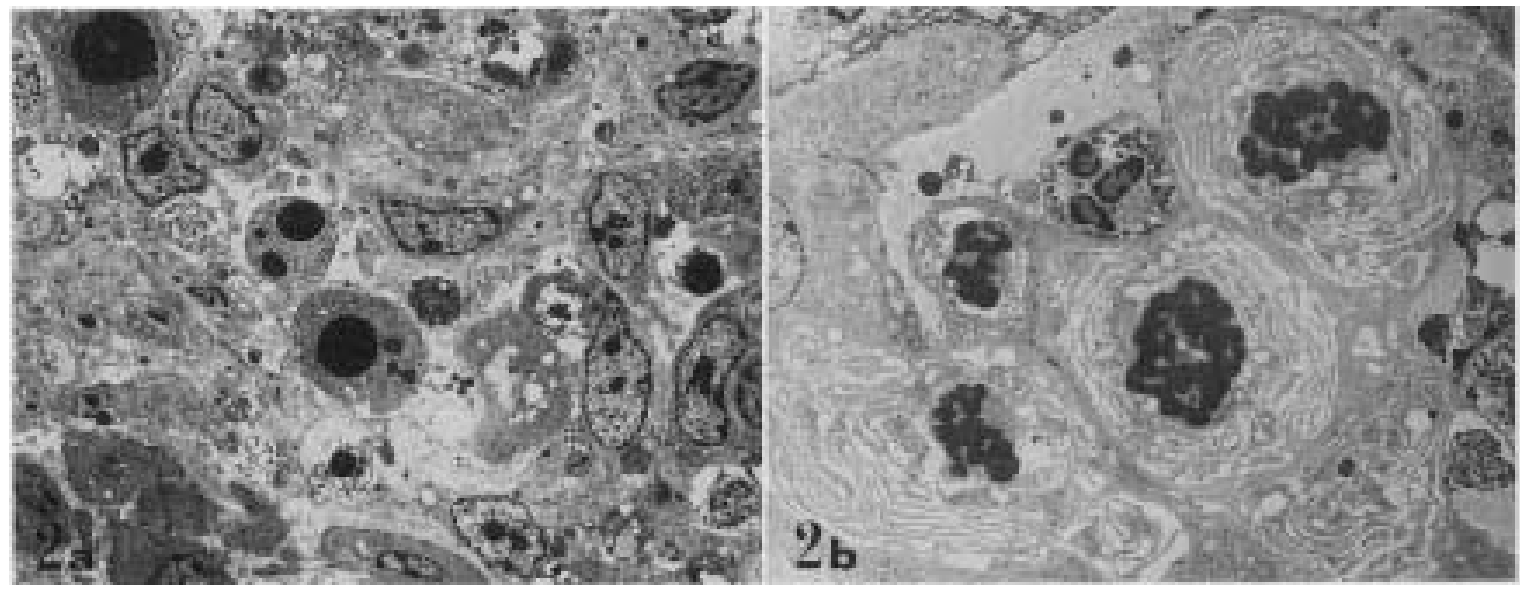

Figs. 2a \& b. Electron micrograph of the small intestine of the intoxicated calf euthanized $30 \mathrm{hr}$ after administration. TEM. Apoptotic cells in the intestinal crypt (a). Note the condensed nuclear chromatin masses in the intestinal crypt. $\times 1,470$. Mitotically arrested cells in the intestinal crypt (b). Note marked dilatation of endoplasmic reticulum and increased density of condensed chromosomal material without mitochondorial abnormality. $\times 1,900$.

has an ability to arrest mitosis [14]. Colchicine has been known to induce apoptosis in vitro and in vivo $[1,3,6,8$, $11,13,15]$. Thus apoptosis observed in this study may have been induced by the cytotoxic effect of ingredient colchicine.

Furthermore, electron microscopy revealed that epithelial cells, which were observed as mitotically arrested cells by light microscopy, had condensed nuclear chromatin masses, swollen cytoplasm without mitochondrial abnormality and dilated endoplasmic reticulum. These cells showed a TUNEL-negative reaction. The epithelial cells with condensed nuclear chromatin masses and dilated endoplasmic reticulum have been observed in the small intestine when administrating MDD such as vincristine to mice $[2,9,10]$. These unique changes have been shown to result from disruption of the mitotic spindle apparatus and arrest of cells in the metaphase caused by MDD [9]. The appearance of such unique changes might be a characteristic finding of MDD toxicosis. In the present study, similar changes were seen in the basal epithelium of the digestive tract of the intoxicated calves, and autumn crocus containing colchicine was shown to induce the unique changes similarly to other MDD. These unique changes differed from apoptosis in TUNEL stainability and ultrastructural findings. Our results suggest that autumn crocus injures epithelial cells of the digestive tract by two different mechanisms leading to apoptotic cells on the one hand, and to mitotically arrested cells on the other hand. The present study confirmed that the pathological changes induced by autumn crocus are associated with morphological features of apoptosis.

\section{REFERENCES}

1. Allen, T. D., Scarffe, J. H. and Crowther, D. 1981. Blood Cells 7: 147-160.

2. Anilkumar, T. V., Sarraf, C. E., Hunt, T. and Alison, M. R. 1992. Br. J. Cancer. 65: 552-558.

3. Bonfoco, E., Ceccatelli, S., Manzo, L. and Nicotera, P. 1995. Exp. Cell Res. 218: 189-200.

4. Brunetor, J. 1995. Phenethylisoquinolines. pp. 771-777. In: Pharmacogrosy, Phytochemistry, Medicinal Plants, Lavoisier Publishing, Paris,

5. Bumbasirevic, V., Skaro-Milic, A., Mircic, A. and Djuricic, B. 1995. Scanning Microscopy 9: 509-518.

6. Ceccatelli S., Ahlbom E., Diana A. and Zhivotovsky, B. 1997. Neuroreport 8: 377-383.

7. Chareyre, S., Meram, D., Pulce, C. and Descotes, J. 1989. Vet. Hum. Toxicol. 31: 261-262.

8. Duncan, A. M. V. and Heddle, J. A. 1984. Cancer Letters 23: 307-311.

9. Harmon, B. V., Takano, Y. S., Winterford, C. M. and Potten C. S. 1992. Cell Prolif. 25: 523-536.

10. Ijili, K. and Potten, C. S. 1987. Br. J. Cancer 55: 113-123.

11. Kaur, C. 1997. Arch. Histol. Cytol. 60. 453-462.

12. Kerr, J. F., Wyllie, A. H. and Currie, A.R. 1972. Br. J. Cancer 26: 239-257.

13. Suzuki, Y., Takeda, M., Obara, N. and Suzuki, N. 1998. Anat. Embryol. 198: 43-51.

14. Takano, Y. and Okudaira, M. 1993. Pathol. Res. Prac. 189: 197-230.

15. Tsukidate, K., Yamamoto, K., Snyder, J. W. and Faeber, J. L. 1993. Am. J. Pathol. 143: 918-925.

16. Wyllie, A. H., Kerr, J. F. R. and Currie, A. R. 1980. Int. Rev. Cytol. 68: 251-306.

17. Yamada, M., Nakagawa, M., Haritani, M., Kobayashi, M., Furuoka, H. and Matsui, T. 1998. J. Vet. Med. Sci. 60: 949952. 